\title{
Microfluidics for bioapplications
}

\author{
Oliver Geschke
}

Published online: 16 August 2009

(C) Springer-Verlag 2009

A few months ago, when I was asked to gather articles for a special issue for Analytical and Bioanalytical Chemistry, I did not hesitate. However, I needed to come up with a good working title matching the readers' interest and my own education. Considering my background, the title should include the term "microfluidics" and something with "bio," and the articles should preferably deal with applied science. Out came the somewhat preliminary title "Microfluidics for bioapplications," which actually ended up being the final one.

Why did I choose this obviously very broad title? Well, first, I wanted to highlight the diversity of applied science in this field. Second, but no less important, to maintain a high quality standard, it is of great importance that a significant number of manuscripts be submitted, from which only the best ones will be selected for publication in Analytical and Bioanalytical Chemistry. A bit of competition usually gives better qualitythe conferences with the highest rejection rates are typically the most attractive ones.

What does the term "microfluidics" now mean in a common understanding? Often people's first impression of a microfluidic system in a bioapplication is a point-of-care device, for instance, a little handheld device analyzing blood samples for a total cell count or to perform glucose measurements. We can relate to those systems, because almost everyone can see the advantage of taking only a small blood sample and most of us are ready to pay a bit extra for our own quality of life.

In this special issue, you will find three review articles dealing with point-of-care analysis - each of them accessing the field from a different angle. One of them focuses on optofluidic technologies, another covers new directions in

O. Geschke $(\square)$

Polymeric Enabling Microsystems (POEM), DTU Nanotech,

Department of Micro- and Nanotechnology,

Technical University of Denmark,

Building 345 East,

2800 Kongens Lyngby, Denmark

e-mail: oliver.geschke@nanotech.dtu.dk medical biosensors using conductive polymer electrodes, whereas the third deals with an overview of miniaturized tools and devices.

Another interesting review article deals with the challenge of increasing the specificity and function of microarrays. This application surely has a very great potential for screening techniques and I am convinced that it will be very interesting reading for most of you.

Whereas "biosensing" in one way or another has been known and used for a long time and certainly has filled many pages in numerous journals, the use of microfluidics for the production of chemically pure substances is not very widespread. By using biomimetic membranes, for instance, one can generate ultrapure water- “just" by mimicking Mother Nature's templates. What Mother Nature has developed and optimized over millions of years now seems possible to use for industrial production - a very exciting topic, I think. The same overall idea of using Nature to produce substances in a controlled way is covered by a review article about microbioreactors in fermentation processes.

So please lean back in your armchair and enjoy your reading.

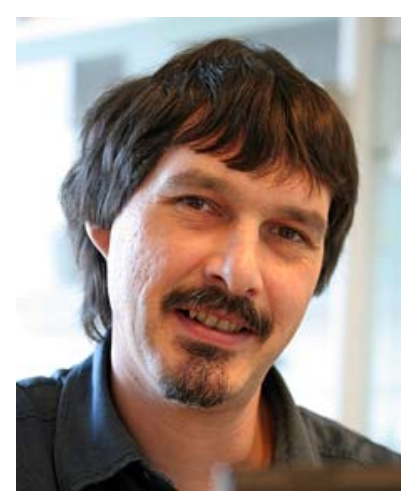

Oliver Geschke is Associate Professor at the Department of Micro- and Nanotechnology (DTU Nanotech) at the Technical University of Denmark. He was educated as an analytical chemist and his research interest is now microfabrication techniques for inexpensive polymer microsystems, mainly for biological and biotechnological applications. Direct prototyping such as laser ablation and micromilling and reproduction methods such as hot/soft embossing and molding in a variety of polymer materials are the techniques commonly applied in his laboratory. Oliver has been the chief editor of the textbook Microsystems engineering of lab-on-a-chip devices and provides courses on this subject at various international conferences. His research group currently consists of one postdoctoral fellow and four PhD students. 